\title{
What can cognitive science tell us about scientific revolutions?*
}

\author{
Alexander BIRD
}

Received: 29.6.2012

Final Version: 30.7.2012

BIBLID [0495-4548 (2012) 27: 75; pp. 293-321]

ABSTRACT: Kuhn's Structure of Scientific Revolutions is notable for the readiness with which it drew on the results of cognitive psychology. These naturalistic elements were not well received and Kuhn did not subsequently develop them in his published work. Nonetheless, in a philosophical climate more receptive to naturalism, we are able to give a more positive evaluation of Kuhn's proposals. Recently, philosophers such as Nersessian, Nickles, Andersen, Barker, and Chen have used the results of work on case-based reasoning, analogical thinking, dynamic frames, and the like to illuminate and develop various aspects of Kuhn's thought in Structure. In particular this work aims to give depth to the Kuhnian concepts of a paradigm and incommensurability. I review this work and identify two broad strands of research. One emphasizes work on concepts; the other focusses on cognitive habits. Contrasting these, I argue that the conceptual strand fails to be a complete account of scientific revolutions. We need a broad approach that draws on a variety of resources in psychology and cognitive science.

Keywords: Kuhn; cognitive science; incommensurability; analogy; naturalism.

RESUMEN: La estructura de las revoluciones cientificas de Kuhn es destacable por la facilidad con que aprovecha los resultados de la psicología cognitiva. Estos elementos naturalistas no tuvieron una buena acogida y Kuhn no los desarrolló posteriormente en su trabajo publicado. No obstante, desde un ambiente filosófico más receptivo hacia el naturalismo podemos ofrecer una evaluación más positiva de las propuestas de Kuhn. Recientemente, algunos filósofos como Nersessian, Nickles, Andersen, Barker y Chen han utilizado los resultados del trabajo sobre el razonamiento basado en casos, el pensamiento analógico, los marcos dinámicos, etc., para iluminar y desarrollar varios aspectos del pensamiento de Kuhn en La estructura. En particular, este trabajo intenta dar profundidad a los conceptos kuhnianos de paradigma e inconmensurabilidad. En este artículo examino dicho trabajo e identifico dos principales corrientes de investigación. Una de ellas subraya el trabajo sobre conceptos y la otra se centra en los hábitos cognitivos. Después de contrastar ambas, sostengo que la corriente conceptual no logra ser una explicación completa de las revoluciones científicas. Necesitamos una perspectiva amplia que aproveche una variedad de recursos de la psicología y la ciencia cognitiva.

Palabras clave: Kuhn; ciencia cognitiva; inconmensurabilidad; analogía; naturalismo.

\footnotetext{
* I am grateful for the comments of audiences in Boston and Loughborough on early versions of this paper, and to an anonymous referee. Research for this project was supported by a Research Fellowship funded by the Arts and Humanities Research Council (Grant $\mathrm{AH} / \mathrm{I004432/1).}$
} 


\section{Introduction}

Thomas Kuhn's Structure of Scientific Revolutions is a work intended to have philosophical significance but which draws most of its resources from history of science. Structure has had enormous influence in both philosophy and history or science. It has also had a great deal of influence in the sociology of science (and social theory more widely). Perhaps surprisingly, for all that Kuhn's ideas were adopted and developed in sociology, Kuhn's own appeals to works in sociology in Structure are few. Rather more numerous are Kuhn's references to works in psychology. Kuhn's interest in psychology was largely ignored in the decades following the publication of Structure. The one exception to the latter concerns Kuhn's remarks drawing on Gestalt psychology, which received a hostile reception from philosophers, with little real attempt to understand what Kuhn was seeking to do with those ideas.

One reason why his philosophical contemporaries dismissed Kuhn's appeal to Gestalt psychology and ignored his discussion of experimental results in cognitive psychology, for example those stemming from the work of Kuhn's Harvard colleagues, Jerome Bruner and Leo Postman, is that such references to the results of empirical science in supporting an argument with philosophical conclusions were unfamiliar in philosophy. While this kind of naturalism is now part of the philosophical landscape, it went against the purely aprioristic grain of philosophy in the 1960s. ${ }^{1}$

However, now we are indeed open to naturalistic approaches, with the work of the sciences playing a part in the construction and assessment of philosophical theses, we should revisit Kuhn's interest in cognitive psychology. We should ask how his theories may be developed and evaluated in the light of research in psychology and cognitive science that has been carried out since the publication of Structure. In this paper I report on two broad ways in which such work has been deployed to develop Kuhnian themes. The first starts with the exemplar idea and argues that training with exemplars can inculcate certain cognitive habits, which may be used to explain the functioning of paradigms in normal science as well as the phenomenon of of incommensurability in revolutionary science. This approach takes its cue primarily from Kuhn's work in Structure. The second draws upon work on concepts in cognitive science; the most advanced work here is that by Hanne Andersen, Peter Barker, and Xiang Chen, drawing upon the work of Lawrence Barsalou on dynamic frames. Because the second approach is focussed on concepts, and because Kuhn's interest in issues of meaning grew after the publication of Structure, that approach draws to a greater extent on Kuhn's later writings. My own view is

${ }^{1}$ Mention of examples from psychology was not itself unprecedented. Hanson's Patterns of Discovery (1958) also does this. But Hanson's illustrative use of psychological cases is different from Kuhn's evidential use. Furthermore, in Kuhn's hands those examples added to the (mistaken) impression that he was promoting an irrationalist picture of science, whereas there was no perception of such an agenda in Hanson's work.

Theoria 75 (2012): 293-321 
that the first approach is potentially the more fruitful. I shall argue that the second approach is rather less comprehensive than it claims in its ability either to articulate Kuhn's theory of scientific revolutions or to understand the revolutions themselves.

\section{Exemplars}

I share Kuhn's view that the idea of an exemplar is the most novel aspect of The Structure of Scientific Revolutions $(1970,187)$. He said that it was also the least well understood, and matters in this regard are a little better now than forty years ago, but not much. The preceding logical empiricist view of scientific cognition is that the process of generating new ideas in science is a matter of creativity and is to be understood by psychology if it can be understood at all; this is the context of discovery. Entirely separate is the context of justification whereby an idea, say a new hypothesis, is evaluated against the evidence. This is the epistemic cornerstone of the scientific method. The relationship is supposed to be an apriori one, and it is the task of philosophers to clarify its details. A good example of this kind of approach is Hempel's deductivenomological account of confirmation: a hypothesis $h$ is confirmed by evidence $e$ in the light of background knowledge of relevant conditions $c$ if and only if $e$ is deducible from $h \wedge c$.

Kuhn's proposal is radically different. First, the relevant unit of assessment is not the hypothesis but is the puzzle-solution. Secondly, the logical empiricists held that the hypothesis is evaluated against (total relevant) evidence, whereas Kuhn holds that the evaluation of a proposed puzzle-solution concerns the relevant evidence, the puzzle itself, and the puzzle-solving tradition from which it comes. Thirdly, whereas the logical empiricists held the evaluation relation to be a logical and apriori one, Kuhn does not think that evaluation of a proposed puzzle-solution is apriori. Indeed, the relationship between puzzle and proposed solution may differ from field to field. How do we assess whether the relationship is a good one? The principal cognitive process involves perceiving similarities between, on the one hand, the package of puzzle-plus-proposed-solution, and, on the other hand, an exemplary package of past-puzzle-plus-its-solution. The exemplary puzzle solution is the paradigm in the narrow sense: a past success held up by the scientific community as a model of how science is done in this field. There are of course questions to be asked about why this exemplary puzzle solution should have that status, to which Kuhn has some answers. But for current purposes, we need to note that what justifies a proposed puzzle solution in the eyes of the community is the perceived similarity between that new puzzle solution and the existing paradigm. Perceiving similarity here is akin to the process of cognition involved in seeing that John looks like his sister Jane, or the ability of a connoisseur to recognize the painter of a painting she has not seen before. These are genuine acts of cognition, but they are not to be understood along the aprioristic lines of the logical empiricists. Here is how 
Kuhn $(1970,189)$ sees this at work in learning science:

[Students] regularly report that they have read through a chapter of their text, understood it perfectly, but nonetheless had difficulty solving a number of the problems at the chapter's end. Ordinarily, also, those difficulties dissolve in the same way. The student discovers, with or without the assistance of his instructor, a way to see his problem as like a problem he has already encountered. Having seen the resemblance, grasped the analogy between two or more distinct problems, he can interrelate symbols and attach them to nature in the ways that have proved effective before.

Connoisseurship in art provides an instructive illustration of the cognitive processes involved in scientific puzzle-solving. The art dealer and historian Bendor Grosvenor (2011) explains,

The ability to tell almost instinctively who painted a picture is defined ... as connoisseurship. The word is derived from the Latin cognoscere, to get to know. The theory is that the repeated study of an artist's work allows one to become so familiar with his or her style and technique that they can be easily recognized, just as we may recognize the author of a letter not from the signature at the end, but from the handwriting at the beginning.

The key here is repeated study. It is by exposure to the works of an artist and their study that one can recognize other works by the same artist. The resulting ability is almost instinctive, by which I take it that Grosvenor means that the knowledge is not mediated by a lengthy process of ratiocination. One can know without having a full appreciation of exactly on what basis one knows. Interestingly, Grosvenor does not think that immediate instinctive response is quite right either:

In 1939 the noted art historian Max Friedlander wrote,"The way in which an intuitive verdict is reached can, from the nature of things, only be described inadequately. A picture is shown to me. I glance at it, and declare it to be a work by Memling, without having proceeded to an examination of its full complexity of artistic form." Unsurprisingly, only about half of Friedlander's attributions have stood the test of time.

Grosvenor thinks that connoisseurship can be supplemented by science, in which case it cannot be an unreflective response. Furthermore, we should note the contrast between Grosvenor's emphasis on study and 'close looking' and Friedlander's 'glance'. Intuition comes about as a result of a deep acquaintance with the exemplar-paintings and careful study of the puzzle-painting. The successful connoisseur will look carefully at the brush-work, the pigments used, the structure of the composition and so forth before coming to a judgment. So while the judgment is almost instinctive, it is different from instinct or intuition in two respects: (i) it is the product of a learned ability, the outcome of prolonged study, and (ii) the judgment may well comes about after reflection, and will be better when it does so.

I suggest that connoisseurship exemplifies the very same kinds of cognitive 
process as Kuhn sees in science. In science the reflective process may be more involved, but ultimately the nature of the judgment is the same, that the target (painting, puzzle-solution) resembles the exemplars. It can be seen again in Kuhn's parallel with crossword puzzles. Sometimes an experienced solver of such puzzles will immediately see a solution on reading the clue, but often the process will require some thought before an answer reveals itself. That it is the correct answer will not be a matter of a logical relation between it and the clue and puzzle (though spotting certain logical relations may be part of the reflective process) and the correctness of the answer will not be readily apparent to someone who lacks experience with such puzzles.

Since this is so far from the traditional epistemology of science and its search for logical relations of confirmation, it is perhaps little surprise that, in Kuhn's view, it is the main source for the controversies and misunderstanding evoked by Structure, and in particular the criticism that he is portraying science as a subjective and irrational enterprise $(1970,175)$. Nonetheless, says Kuhn, the tacit knowledge embedded in exemplars, 'though [it is not], without essential change, subject to paraphrase in terms of rules and criteria, it is nevertheless systematic, time tested, and in some sense corrigible'.

The fact that we do spot similarities between family members, that art connoisseurs do get to know almost instinctively who painted a newly discovered picture, and so forth shows that there are indeed mechanisms of human cognition that meet Kuhn's description of those involved in science. Furthermore, artificial neural networks have been developed that embody learning with exemplars and have high levels of success in cognitive tasks such as face and speech recognition, diagnosis in medicine, spam filtering and so forth. So the question cannot be, 'is such cognition possible?' or even 'would science be irrational if it were to involve such cognition?' For such cognition does exist and it would be bizarre to label high levels of success (e.g. in recognising your children) are 'irrational'. Rather, the important question is, does science really involve such processes?

Let us look then, albeit briefly, at the evidence for a central role for Kuhnian pattern recognition in scientific cognition. One piece of evidence is that already referred to by Kuhn in the quotation above. Exercises in textbooks are designed to assist students to recognise certain puzzle situations as demanding solutions using certain equations or other techniques exemplified by worked examples in the text. The first questions are straightforward, being most similar to those exemplars. Later questions are increasingly difficult, principally by being less immediately similar to the exemplars. Working through the questions will provide the student with a trained sense of when a problem will call for a certain kind of solution or approach. An experienced student or an expert will see immediately that a puzzle requires these equations to be deployed in this way; a neophyte may know those equations but not have any idea about how they are to be used in solving these puzzles. This plus the fact that skill in this regard is a matter of degree that is improved by repeated practice suggests that this is indeed an ability much like pattern recognition and not a matter of de- 
ploying some general problem-solving capacity. Furthermore, experts are able to see problems as exemplifying deeper physical patterns (e.g. as requiring application of a certain principle, such as least action) while neophytes see only superficial patterns (e.g. classifying puzzles by the kinds of entity involved) (Chi, Feltovich and Glaser 1981), conforming to Kuhn's claim that training with exemplars induces new similarity spaces. Howard Margolis (1987) makes an extended and persuasive case for the centrality of pattern recognition in all judgment, including science, supported by historical case studies.

Kuhn's hypothesis concerning reasoning with paradigms sees scientific cognition as an instance of analogical reasoning. The scientist learns to sees an analogy between her puzzle and the paradigm puzzle and so see how a solution to the latter might be transformed to provide a solution to the former. Analogical reasoning of this sort is indeed ubiquitous in science, as is shown by close studies of scientists using the approaches of psychology and anthropology (Holyoak and Thagard 1995, 1997, Dunbar 1996, 1999, Gentner, Holyoak and Kokinov 2001) as well as historical research on past episodes of scientific change (Margolis 1987, Gentner and Jeziorski 1993). Such studies not only reveal that analogical reasoning is central to scientific thinking but also show that there are different kinds and depths of analogy that are deployed for different purposes.

Of particular interest is the work done on Case-Based Reasoning (CBR). According to CBR, a case-based reasoner employs a stock of concrete cases; when a new problem comes along, she compares the new case to the past stock. Analogies between the new cases and certain of the stock cases will prompt analogous solutions. Some analogies may be stronger than others, making the corresponding analogical solutions more plausible than the other possibilities. CBR has been of primary interest to 'knowledge engineers', i.e. those building artificial intelligence systems to solve certain kinds of problem; the fact that such models are efficacious in solving scientific and other problems is indirect evidence for the Kuhnian thesis. Thomas Nickles (2003) is, as far as I am aware, the first to make the connection between Kuhnian exemplars and CBR. Nickles does note aspects in which the two diverge. CBR typically includes negative cases, i.e. cases where an analogy fails, which can often be instructive, whereas Kuhn's exemplars are all positive cases. Secondly, Kuhn does not say enough about the historical development of exemplars. This is an important point, for while Kuhn talks of Principia Mathematica as a paradigm, he also tells us that students learn the paradigm through exemplars in textbooks and the exercises whereby they learn to apply the exemplars and to see different puzzles as belonging together. But the exemplars of classical mechanics found in textbooks are not Newton's exemplars in Principia. The exemplars have themselves undergone a process of historical development, one, according to Nickles, whereby we do not just fit new puzzles to old exemplars, but the exemplars themselves change in response to the new puzzles. Nickles regards these divergences as exhibiting shortcomings in Kuhn's account. But the central significance of exemplars and the insight that CBR may explain how they operate remain. Indeed, the naturalistic nature of Kuhn's claims, made before 
much of the current evidence became available, implies that we should expect Kuhn's picture to be modified as further evidence accrues.

The feature of CBR I wish to emphasize is one that is in fact ubiquitous in our cognitive lives; it is the significance of cognitive habits. While Hume and others were wrong in thinking that associationism (or classical conditioning) could explain everything about the way we think, it can nonetheless play a significant role in explaining many things. We become adept at playing a piece of music through exercise, so that certain fingering that needed conscious thought initially is now performed with unconscious fluency. The same can be true of intellectual activity also. Indeed Kuhn likens the practice students get (SSR 47) in working through scientific exercises to finger exercises. At first it will require hard thought and perhaps some trial and error attempts to see how a particular theory should be applied to a new puzzle. In due course the student will find that she has some facility in applying the theory to new puzzles that may be of the same class as ones she has encountered before. It is only a difference of degree for the great scientist, as Kuhn tells us:

Scientists model one problem solution on another, often with only a minimal recourse to symbolic generalizations. Galileo found that ball rolling down an inclined place acquires just enough velocity to return to the same vertical height on a second incline of any slope, and he learned to see that experimental situation as like the pendulum with a point mass for a bob. (Kuhn 1974, 305)

Kuhn goes on to say that Huyghens's solution to the problem of the centre of gravity of physical pendulum is modelled on Galileo's point pendulum, and then that Bernoulli's account of water-flow from an orifice in a storage tank resembles Huyghens's pendulum.

So the connection between a theory and a puzzle is one that starts out as obscure and difficult to see but eventually becomes second nature. 'Second nature' is so-called because it is, to its possessor, entirely naturally and intuitive, the reactions are instinctive. On the other hand it is 'second'-acquired, not innate. Such connections I have called 'quasi-intuitive connections'. Such connections cause us to make inferences, e.g. that a certain puzzle-situation can be seen as a case of simple harmonic motion. It is natural to use perceptual terms in such cases: as Kuhn says, Galileo sees the ball on the inclined plane as like the pendulum: on seeing the ball Galileo quasi-intuitively infers that what is true of the pendulum is true of the ball; that analogy (second) naturally springs to his mind. In many such cases the nature of the subject's total experience is the effect, in part, of the learned associations, the quasi-intuitive inferences the subject makes. Importantly, is this experience that the subject reports as an observation, as data:

In The Structure of Scientific Revolutions, particularly chap.10, I repeatedly insist that members of different scientific communities live in different worlds and that scientific revolutions change the worlds in which a scientist works. I would now want to say that members of different communities are presented with different data by the same stimuli. Notice, however, that that change does not make phrases like "a 
different world" inappropriate. The given world, whether everyday or scientific, is not a world of stimuli. (Kuhn 1974, 309)

I have elsewhere argued that one way to understand Kuhn's 'worlds' and 'world-changes' is in terms of the shared quasi-intuitive connections of a scientific community (Bird 2005). Certain quasi-intuitive connections, because instilled by training with exemplars, are second nature to all members of the community. When exemplars change, these patterns of quasi-intuitive connections change: inferences that were permitted before are not not permitted, and vice-versa. To take a simplified example, an Aristotelian is permitted to infer from ' $x$ is in motion', to 'there is a cause of $x$ 's motion', whereas the Newtonian is not permitted to make that inference; for a Newtonian, only the following is permitted ' $x$ has changed its motion (i.e. accelerated or decelerated)' therefore 'there is a cause of $x$ 's change in motion'. This is the same transition in quasi-intuitive connections that students have to make when learning physics.

I also propose that such changes in patterns of quasi-intuitive connections can also account for incommensurability (2007). When one author employs quasi-intuitive connections that are not possessed by a reader, then it will be very difficult for the reader to make sense of author's reasoning. It will appear to be full of non-sequiturs and so lacking in rationality. Deeper acquaintance with the author and the author's exemplars may eventually allow the reader to understand the tacit connections the author is making and so be able to rationalise the author's discussion. I conjecture that something like this explains Kuhn's experience on initially finding Aristotle to be an incomprehensibly bad physicist then converted to appreciating his genius, an experience that was formative in Kuhn's approach to incommensurability in Structure (1970, v; 1977, xi-xii; 1987, 8-9). I believe that this way of understanding incommensurability can also help us appreciate the incommensurability between an old paradigm and its replacement. Because the quasi-intuitive connections are deeply ingrained in those practising in the old paradigm, it is difficult for them to grasp that they are even employing those connections and so difficult also to give them up. That will be most true for those who have worked most extensively in the old paradigm, i.e. older scientists and those working centrally, and explains why younger scientists and those who come from outside the specialty are able to see possibilities that are in effect ruled out by the quasi-intuitive connections.

The proposals I sketch above are in need of further empirical confirmation. Yet, the fact that they rest upon a basis of extensive research in psychology, cognitive science, and artificial intelligence, as well as history of science, lends them plausibility. From the perspective of the remainder of this paper, the important feature to bear in mind is that the central explanatory tool is: a set of cognitive habits learned by training with exemplars. This contrasts with the centrality of conceptual structures in the alternative approach to understanding Kuhn in relation to cognitive science that I turn to now. 


\section{Dynamic frames}

Some of those who believe that cognitive psychology can assist in illuminating the problems with which Kuhn was grappling focus on conceptual change. Nancy Nersessian's 'cognitive-historical' approach is a leading example (Nersessian 1987, 1992, 2003). Hanne Andersen, Peter Barker, and Xiang Chen have developed related ideas in detail to produce a sophisticated account of conceptual change that draws upon cognitive science and which vindicates what they take to be a Kuhnian approach to incommensurability and scientific revolutions (Andersen, Barker and Chen 1996, 2006; Chen, Andersen and Barker 1998). While acknowledging the significance of these ideas, and accepting that they may illuminate aspects of incommensurability, I am sceptical regarding the central place given to specifically conceptual change. Because the approach of Andersen, Barker, and Chen (henceforth $\mathrm{ABC}$ ) is more exclusively conceptual, it is on their work that I concentrate in this section.

According to ABC (2006, 5), 'Between 1969 and 1994, Kuhn elaborated an account of scientific change in which the theory of concepts holds a central place.' Andersen, Barker, and Chen (henceforth ABC) argue that Kuhn's account built on ideas from Wittgenstein about concepts, in particular the family resemblance idea, that he had introduced before this period. They say that these Wittgensteinian ideas were 'almost universally repudiated by philosophers in the English-speaking world', who preferred the classical definitional account of concepts. Nonetheless, the approach of Wittgenstein and Kuhn received empirical confirmation, first in the work of Eleanor Rosch and her colleagues (Rosch 1973, Rosch and Mervis 1975, Rosch 1988).

The classical theory of concepts says that a concept is a structured entity, where that structure consists of a set of conditions, individually necessary and jointly sufficient for the correct application of the concept. While versions of the classical theory can be traced back to Plato, and a more recent version to Locke, the classical view was central to logical empiricism. Propositions are either synthetic or analytic. The truth of the former is verified by empirical procedures. The truth of the latter is verified by decomposing the constituent concepts into their components, which are the necessary and sufficient conditions for their correct application; a true analytic proposition will be revealed to be a tautology. Since a large range of non-empirical (but not nonsensical) propositions, including those of philosophy and mathematics, were held to be analytic, logical empiricism's commitment to the classical view of concepts is significant.

Wittgenstein's later philosophy challenged the classical view. In particular, the fact, as he insisted, that some concepts are family resemblance concepts appeared to refute the idea that the correct application of a concept is determined by a set of necessary and sufficient conditions. A number of entities might fall under a family resemblance concept yet share no relevant property in common; so no (non-trivial) property is individually necessary. What makes the entities fall under the concept is the fact that those entities are related 
by a network of different resemblances, like the resemblances between various members of a family.

According to ABC, the classical view, although dominant, came under pressure not only from Wittgenstein's alternative, later followed by Kuhn, but also from the fact that successful analyses of concepts into necessary and sufficient conditions were few: many crucial concepts (such as KNOWLEDGE) resisted formidable efforts to analyse them. Most importantly, the classical view, it is alleged, is refuted by the empirical work of Rosch and others in the 1970s.

This empirical work shows that concept users regard some instances of a concept as more typical than others, even when the instances all fall under the concept. For example a sparrow is held to be a more typical instance of BIRD than a chicken. According to the classical view both sparrows and chickens satisfy the necessary and sufficient conditions for BIRD; the concept makes no distinction between them. Such typicality effects usually show a graded structure, a structure which is revealed in certain kinds of performance, such as speed in categorising entities. These empirical results led to the development of an alternative to the classical account of concepts, the prototype view.

The prototype account of concepts is similar to the classical view in that it regards concepts as structured, consisting of a list of features. However, these features are not necessary features, features possessed by all instances of a concept. Rather such features are weighted, reflecting the fact that items in the concept's extension tend to have these features. Such weights, which may be thought of in statistical terms (possibly reflecting frequency in the extension), will allow there to be a relation of similarity between the representation of some entity and the concept, a relation that comes in degrees. So BIRD may include the feature list (or prototype) HAS WINGS, IS FEATHERED, LAYS EGGS, HAS A BEAK, SMALL, SINGS, FLIES, NESTS. The weighting of these features means the following: sparrows are more similar to the prototype than chickens, because there is some weighting attached to SMALL and SINGS; both sparrows and chickens have sufficient similarity to the prototype to be regarded as birds; while SMALL and SINGS do contribute to sparrows being classified as birds their absence from chickens does not disqualify chickens from the category (they are not necessary conditions).

The prototype account seems to allow for family resemblance concepts: features can be relevant to classification without being necessary conditions; similarity is the basis of classification, but not all instances of the concept are similar in the same way. Rosch herself assimilated Wittgenstein's view to the prototype theory she developed. ABC link both to Kuhn's view of concepts. Kuhn does refer to Wittgenstein's family resemblance idea over a page and a half in Structure. ${ }^{2}$ ABC draw on this and on Kuhn's discussion in 'Second thoughts on paradigms' (Kuhn 1974), where Kuhn describes a parent teaching a child to distinguish ducks, geese, and swans. Initially the child sees the dif-

${ }^{2}$ Below I shall argue that Kuhn's reference to Wittgenstein is incidental, not central to Structure. 
ferences between individual swans as just as salient as the differences between swans and geese. The parent then trains the child by pointing out which of the birds they encounter are ducks, geese, and swans, and also by affirming or correcting the child's own attempts at classification. Now the child can group the animals correctly, and thereby gets to know what 'duck', 'goose', and 'swan' mean. ABC point out that Kuhn generalizes this to other scientific concepts. It is by learning the similarities between different applications of the law sketch $\mathbf{F}=m \mathbf{a}$ to concrete problem situations that a student learns the concepts of force and mass. 'A conceptual structure,' they say, 'is established by grouping problem situations into similarity classes corresponding to the various expressions of the law sketch' (Andersen, Barker and Chen 1996, 31).

$\mathrm{ABC}$ go beyond the prototype theory to a development from the same set of ideas, Lawrence Barsalou's dynamic frame account. The frame account in effect adds structure to the prototype theory. As with the prototype theory we identify a concept with various features, which we do as follows. A superordinate concept is associated with several attributes, for example BIRD with BEAK, NECK, COLOUR, SIZE, and GAIT. Each attribute may take one of a number of values, e.g. BEAK may take the values ROUND or POINTED and FOOT may take the values WEBBED or CLAWED. A particular subordinate concept is identified with specific values of these attributes: WATER BIRD has the values ROUND for BEAK, WEBBED for FOOT while LAND BIRD has the values POINTED for BEAK and CLAWED for FOOT. An important property of Barsalou's frames is that there can be connections between components of the structure. For example, one might note that there is a correlation between beak shape and foot type: birds with webbed feet have round bills and birds with claws have pointed beaks. Such correlations are part of the conceptual structure. ABC $(2006,209)$ make it clear that in their view (and in Kuhn's view) 'there is no distinction between defining and contingent features of an object', so all beliefs about a kind of object are represented by some aspect of the conceptual structure, including such such connections ('constraints').

As indicated with the example of the superordinate concept BIRD and the subordinate concepts WATER BIRD and LAND BIRD, we can use the frame account to understand taxonomic hierarchies. Such hierarchies are governed by three principles: the no-overlap principle: distinct concepts do no partially overlap (either they do not overlap at all or one concept is subordinate to the other); the exhaustion principle: when a superordinate concept has subordinate concepts, every entity falling under the superordinate concept falls under some subordinate concept (nothing is left unclassified by the subordinate concepts); the inclusion principle: everything falling under a subordinate concept falls under its superordinate concept.

ABC use the dynamic frame account of concepts to articulate and develop key Kuhnian ideas: anomaly, revolution, and incommensurability. An anomaly occurs when an entity (often a thing but may be an event) is discovered whose classification demands violation of some hierarchical principle. (Because the structures governed by those principles embody our expectations about what 
there is and what it is like, such entities are unexpected and may be difficult to recognise as such.) For example, the South American screamer has webbed feet and a pointed beak. So, on the basis of its beak it seems to require classification under LAND BIRD whereas its webbed feet would propose classification user WATER BIRD. However, to classify the screamer as both LAND BIRD and WATER BIRD would be to violate the no-overlap principle.

If such anomalies are to be accommodated (rather than simply excluded), then the conceptual structure needs to be reformed. As ABC explain, further attributes of BIRD, such as PLUMAGE and TARSUS become relevant so that there can be three exhaustive but mutually exclusive subordinate concepts NATATORES, GRALLATORES, and GALLINAE, where there were previously only two (LAND BIRD and WATER BIRD). Such revisions of the conceptual structure, where existing entities are redistributed in ways that were forbidden by the previous taxonomy, are definitive of scientific revolutions, which $\mathrm{ABC}$ go on the illustrate with more sophisticated examples, such as nuclear physics in the 1930s and the development of the Copernican revolution. ABC point out that this approach allows for revolutions that differ in scale. Revolutionary changes to taxonomic conceptual structures will involve changes to the similarity and difference relationships that define our categories. For example, in the BIRD case, the basis on which similarity and difference between birds has changed; in particular new attributes have been added that contribute to determining the similarity space that were previously irrelevant (e.g. PLUMAGE). Such changes explain incommensurability. $\mathrm{ABC}$ argue that incommensurability does not automatically imply communication failure and use the frame approach to describe different kinds of conceptual change and their consequences.

\section{Discussion}

$\mathrm{ABC}$ have done an important and useful service in articulating a framework for understanding Kuhn's later, taxonomic, account of incommensurability (Kuhn 1987, 1991, 1993. C.f. Sankey 1998). And if Barsalou's account of concepts is largely correct for at least some concepts, then they have also provided an insight into how - in some scientific cases - there can be incommensurability and thereby shed light on the nature of some scientific revolutions, those in which a revolutionary change is centred on a radical rearrangement of taxonomic structure. In this section I will argue that we should see ABC's approach, insightful though it is, as restricted in scope, both as an articulation of Kuhn's theory of scientific revolutions, and as an account of the phenomena of scientific revolutions.

\subsection{Understanding the historical context}

$\mathrm{ABC}$ overstate the case for the dominance of the classical account of concepts among English-speaking philosophers, and for Kuhn's being special in rejecting it for an account along Wittgensteinian lines. Views of concepts inconsis- 
tent with the classical account were widely discussed, for example W. B. Gallie's (1955) idea of an essentially contested concept, Dummett's (1991) prooftheoretic semantics, Quine's meaning nihilism (1951, 1960), Schlick's (1918) notion of implicit definition, developing ideas from Hilbert, the holism of the double-language model of Carnap (1956) and Nagel (1961), and finally the New Theory of Reference (Marcus 1961, Kripke 1971, 1980), probably the dominant current view. Many philosophers articulated Wittgenstein-inspired approaches to concepts, in many case using Waismann's idea of open-texture, for example Hart (1961) in philosophy of law, MacIntyre (1973) in social philosophy, Weitz (1956) and Mandelbaum (1965) in aesthetics, and von Wright (1963) in ethics.

Against such a background, Kuhn's brief discussion of Wittgenstein does not stand out. Many philosophers had a rather deeper engagement with Wittgensteinian ideas, which were widely discussed. And as I shall go on to argue, the latter were not especially important for Kuhn.

At the same time, other views of meaning and of concepts were developed that challenged the classical view. So even if $\mathrm{ABC}$ were correct that what they hold to be a Wittgenstein-Kuhn account of concepts is superior to the classical view, that would not show that the former is our best theory. For there are alternatives out there; and in particular, I suggest, accounts of concepts need to be taken seriously that are consistent with the New Theory of Reference accounts such as atomism that contradict both the classical account and the alleged Wittgenstein-Kuhn account.

\subsection{Understanding Kuhn's theory}

ABC tell us, 'We will show that all of the important features of Kuhn's model may now be seen as consequences of this fundamental account of human concepts and its dynamics'; 'We will elaborate the notion of incommensurability, the central theme of Kuhn's theory of scientific revolutions' (ABC 1998, 6).

Because incommensurability was so contentious and because Kuhn spent so great a proportion of his later work in adjusting and refining his account of incommensurability, it is easy to gain an exaggerated picture of its significance in Structure. Kuhn uses the terms 'incommensurable' and 'incommensurability' only nine times in the first edition of Structure, which contrasts with the hundreds of uses of 'paradigm'. Incommensurability simply is not 'the central theme of Kuhn's theory of scientific revolutions' as that theory is articulated in its locus classicus.

Nor is Kuhn's use of ideas from Wittgenstein in Structure central to that theory. Kuhn completed a draft of Structure around April 1961, i.e. only a few months before completion of the final version as published in 1962 (HoyningenHuene 2006). The principal difference between this draft, now known as ProtoStructure, and Structure is that the latter has a chapter, 'The Priority of Paradigms', that Proto-Structure lacks. Furthermore, the preceding chapter of Structure, entitled 'Normal Science as Puzzle-solving' exists in Proto-Structure as a chapter entitled 'Normal Science as Rule-Determined.' What we may infer 
from these facts is that the material in 'The Priority of Paradigms' is not essential to the basic ideas of Kuhn's theory of scientific revolutions, all of which is in place in Proto-Structure. The brief passage concerning Wittgenstein is in this late additional chapter. And so Wittgenstein-inspired ways of thinking cannot be central to Kuhn's theory of scientific revolutions, contrary to the thrust of ABC's argument. ${ }^{3}$ We may also infer that Kuhn's thinking about rules must have undergone some change after completing Proto-Structure. It is possible that Kuhn showed Proto-Structure to his colleague Stanley Cavell, who pointed him in the direction of Wittgenstein as someone who had something relevant to say about rules, and that Kuhn revised his opinion in response to reading Wittgenstein. Alternatively, Kuhn independently was rethinking the nature and significance of rules, and reading Wittgenstein, again probably at the prompting of Cavell, helped him articulate his new ideas. ${ }^{4}$

Either way, what is important is that the newly added reference to Wittgenstein does not come at a point where Kuhn is dealing with concepts but in a chapter where he is concerned with the more general phenomenon of rules and how they relate to working within a paradigm. 'Normal Science as Puzzlesolving' emphasises the analogy between normal science and puzzle-solving, and a central part of that argument involves showing that like games (including games of puzzle-solving), normal science is played according to rules (as the title of this chapter's original in Proto-Structure emphasizes). But in 'The Priority of Paradigms' Kuhn accepts that this cannot be all there is to working within a paradigm. He points out that a historian seeking the shared rules of a scientific tradition will meet with partial success but also frustration. That is because there can be agreement on what the exemplars are without any explicit, shared articulation of what specific features of those exemplars explain their continued success. But then there is a puzzle about how there can be this agreement without there being a full set of rules that the community are agreed on following. It is in this context that Kuhn includes a footnote to Polanyi's notion of tacit knowledge, for part of the answer is that the agreement is tacit, and not articulated explicitly. Still, that would leave unanswered the question of how this tacit knowledge and tacit agreement come about.

${ }^{3}$ ABC $(2006,105)$ mention Wittgenstein's use of the duck-rabbit and say that Kuhn took over Wittgenstein's examples. But this seems unlikely, since Kuhn mentions the duckrabbit in Proto-Structure and so before he saw the relevance of Wittgenstein's work. The duck-rabbit, first used in psychology by Jastrow (1899), has appeared in psychology textbooks since 1922. It is more probable therefore that Kuhn's examples came from his own interest in Gestalt psychology (which Wittgenstein also had), which, as ABC do note, precedes his acquaintance with the work of Wittgenstein.

${ }^{4}$ It should be noted that there is another tradition in Kuhn scholarship that sees a strong influence by Wittgenstein on Kuhn, for example Kindi (1995a,b), Sharrock and Read (2002), Narboux (2003). Read (2005) rejects the naturalistic approach that is common ground to those discussed in this paper; indeed he regards the use of cognitive science by Nersessian and ABC as un-Wittgensteinian, despite their references to Wittgenstein. In my view both groups exaggerate the significance of Wittgenstein for Kuhn.

Theoria 75 (2012): 293-321 
The passage on Wittgenstein comes next, for it does answer that question. One might think that the application of concepts is governed by explicit rules, and while that may be true up to a point, Wittgenstein's argument surrounding family resemblance concepts shows that this cannot be the whole story. As Kuhn (1970, 45) says, 'For Wittgenstein, in short, games, and chairs, and leaves are natural families, each constituted by a network of overlapping and crisscross resemblances.' It is the importance of learning to spot resemblances that Kuhn identifies here and which replaces the explicit following of rules. This he exploits in the next paragraph when he returns to science:

\begin{abstract}
Something of the same sort may very well hold for the various research problems and techniques that arise within a single normal-scientific tradition. What these have in common is not that they satisfy some explicit or even some fully discoverable set of rules and assumptions that gives the tradition its character and its hold upon the scientific mind. Instead, they may relate by resemblance and by modeling to one or another part of the scientific corpus which the community in question already recognizes as among its established achievements.
\end{abstract}

As this context shows Kuhn is not interested here in articulating a theory of concepts. Rather he is articulating a theory of how learning to recognise resemblances can replace the explicit following of rules. Wittgenstein's point of about concepts is an analogue to Kuhn's point about working with exemplars, albeit one underpinned by the same cognitive ability in recognising patterns of resemblances.

The conclusion of the preceding paragraphs is this. The reference to Wittgenstein in Structure is not central to this theory of scientific revolutions; it is a late addition to that theory. And Kuhn's purpose in talking about Wittgenstein is not to articulate a theory of concepts; it is to show how recognition of resemblances can replace explicit following of rules; and the purpose of that is to give a more satisfactory account of what is involved in working in a paradigm. Furthermore, the reference to Wittgenstein comes nowhere near Kuhn's discussion of incommensurability, which does not make an appearance for another hundred pages. Consequently, we should not think that because Kuhn refers to Wittgenstein in Structure that he is there beginning to develop a Wittgenstein-inspired theory of concepts that is central to his theory of scientific revolutions.

Because it concerns Structure, published in 1962, what I have said so far in this section is consistent ABC's key claim that the theory of concepts is central to Kuhn's account of scientific change elaborated between 1969 and 1994. If they are right, then Kuhn developed a second account of scientific change, substantially different from the theory in Structure. Kuhn does write 'Violation or distortion of a previously unproblematic scientific language is the touchstone for revolutionary change' $(1987,21)$ in his paper 'What are scientific revolutions?', written in 1981. And he does indeed develop a novel account of incommensurability, one based on taxonomic violation, elements of which are found in 'What are scientific revolutions?' Nonetheless, the textual evidence 
for a new theory of scientific change is thin. 'What are scientific revolutions?' is mostly taken up with descriptions of three episodes of revolutionary change, followed by only three pages of discussion. The latter picks out three common features: (i) the changes are locally holistic: several scientific commitments (theoretical claims, etc.) are changed together, where it would make no sense to make the changes piecemeal; (ii) there are changes to the taxonomic categories involved; and (iii) 'a central change of model, metaphor, or analogy', which Kuhn thinks is 'probably the most consequential' of the characteristics (1987, 20 ). While the centrality of of taxonomic change is certainly new, Kuhn says too little, here or elsewhere, to attribute to him a new account of scientific change. At most what we get is a change in what he takes to constitute a scientific revolution. But that leaves untouched the dynamics of scientific revolutions (why they occur, what happens when they occur, and how they are resolved).

Another question for ABC's thesis concerns the timing of the claimed shift in Kuhn's thinking about scientific change. They date is back to 1969, when Kuhn wrote the Postscipt to the second edition of Structure. While this does show important new ideas and emphases, Kuhn is clearly most concerned to clarify and elaborate the central ideas of Structure, those concerning paradigms, exemplars in particular. Crucially, the period they refer to includes Kuhn's 'Second thoughts on paradigms' (1974), which is central to their case that Kuhn held a Wittgenstein-inspired account of concepts - yet Kuhn does not mention Wittgenstein at all in 'Second thoughts'.

Pace ABC, in 'Second thoughts on paradigms' Kuhn does not expound a theory of concepts (he doesn't use the term 'concept' and only sparsely talks about 'meaning'). Rather, he is principally concerned to further articulate his notion of exemplar as paradigm (as the title hints) and to argue that exemplars can function without rules. In particular we do not apply exemplars and their symbolic generalisations to the world by obeying correspondence rules (as the logical positivists would have); rather we do so in virtue of having learned similarities between the exemplary puzzle situation and the puzzles we are confronted with (as discussed in section 2.). If the correspondence rule approach were right, then such a rule might say something like, 'apply Ohm's law to situations with features $\mathrm{F}$ in such-and-such a way', implying that we would have some prior grasp on what $\mathrm{F}$ is. In denying the work supposedly done by correspondence rules, Kuhn denies that we are able to group puzzlesituations by their being F. So how do we know when to apply Ohm's law? Kuhn $(1974,308)$ therefore says, 'I now want to argue, there is a means of processing data into similarity sets which does not depend on a prior answer to the question, similar with respect to what?' But he does not want to deploy scientific examples because 'inevitably the latter prove excessively complex' $(1974,309) .^{5}$ That is why he uses the story of Johnny learning to differentiate

${ }^{5} \mathrm{ABC}$ state that this refers to the learning of concepts. But it is clear from the context provided by the preceding three paragraphs that Kuhn $(1974,308)$ is primarily concerned with 'learning to see two problems as similar' (my emphasis). 
ducks, swans, and geese, because that is a process whereby someone learns to group entities (in story: wildfowl; in science: scientific problems) into classes of similar entities (in story: e.g. ducks; in science: e.g. problems requiring application of Ohm's law). ${ }^{6}$ So although Kuhn's discussion in 'Second thoughts on paradigms' provides fuel for a theory of concepts, developing such a theory is not Kuhn's focus, which he himself tells us is the wider question of the operation of paradigms (understood as exemplars) and their dependence on the process of learning to apprehend similarities by training (rather than rules). ABC's primary source for what they call 'Kuhn's theory of concepts' is not intended to be any such thing (just as Kuhn's reference to Wittgenstein is not intended to articulate a view about meaning). ${ }^{7}$

In summary, Kuhn was not concerned to develop a theory of concepts in Structure; his mention of Wittgenstein there is not central to his argument and is not concerned with promoting a Wittgensteinian view of meaning. And since the theory of concepts does not play a central role in his exposition of the theory of scientific revolutions in Structure it is implausible, in my view, that more advanced theories of concepts provided by cognitive science will illuminate or add to what Kuhn intended in that theory. So ABC's argument depends on there being a later theory of scientific change, that does have a theory of concepts at its core. Yet the evidence that Kuhn developed such a theory is thin. There is no new theory in the Postcript 1969 to the second edition of Structure, nor is there in 'Second thoughts on paradigms' (1974). $\mathrm{ABC}$ interpret the latter as proposing a view of concepts, but closer examination does not support that view. It is true that Kuhn does later develop his ideas concerning incommensurability, and in particular the taxonomic account (1987, 1991, 1993). I fully agree that ABC's approach is a very productive way of developing Kuhn's thoughts in this respect (although I think there are limitations both to the dynamic frame account of concepts and to the taxonomic account of incommensurability). Does the development of that account amount also to a new, revised theory of scientific change? I have argued that the evidence is thin. In any case, as I shall go on to argue, insofar as Kuhn did reconceive revolutions as a certain kind of taxonomic change, the result is an unsatisfactory account of scientific change.

\footnotetext{
${ }^{6}$ Note reference above to Chi, Feltovich and Glaser (1981) and their work in showing how expertise causes changes in which scientific problems are held to be similar.

7 It is also worth noting that Johnny's learning to differentiate waterfowl by creating a mental space of similarities and dissimilarities does not mean that Johnny's concepts DUCK, SWAN and GOOSE are family resemblance concepts, since nothing in the story suggests that the concepts are constituted by criss-crossing resemblances such that no single resemblance is shared by all of one kind. Not all resemblance is family resemblance. The conjecture that Kuhn recognised that fact would explain why he did not refer to Wittgenstein in his discussion. If, as ABC claim, this discussion is a development of his theory of concepts based on the earlier adoption of Wittgenstein's family-resemblance in Structure, then that omission is surprising.
} 


\subsection{Understanding scientific revolutions}

Irrespective of whether we can develop Kuhn's theory within a framework taken from Rosch and Barsalou, is it in fact the case that their theories can tell us something about the phenomena of scientific revolution and incommensurability? Here I argue that there are potentially significant limitations to the scope of the application of those theories. First, we must recognise that there are important objections to those theories that mean that at best they offer only a partial account of concepts. (I shall concentrate on the prototype account, since this is the core of the approach that $\mathrm{ABC}$ adopt.)

The empirical results of the work of Eleanor Rosch are widely (but not universally) agreed to be inconsistent with the classical theory, and so are taken by ABC to support what they regard as the Wittgenstein-Kuhn approach and subsequent developments by Barsalou and others. In the light of the preceding paragraphs we should be wary in inferring from the falsity of the classical theory to the correctness of the 'Wittgenstein-Kuhn approach' - there are other competitors to be considered. Indeed Laurence and Margolis (1999) list five competing types of theories of concepts: the classical theory, the prototype theory, the neo-classical theory, the theory theory, and atomism. All have their problems and all have things to be said in their favour.

It is worth being aware of some of the limitations of the prototype theory:

- The problem of compositionality. Compound concepts are composed of their component concepts. But the prototype of PET FISH is a small, gold animal that lives in a bowl or tank. This cannot be composed from the prototypes for PET (furry, mammalian) and FISH (brown, medium sized, lives in the sea).

- Conceptual ignorance. A subject may have distinct concepts RUTHENIUM and RHODIUM yet be sufficiently ignorant that he has no knowledge that distinguishes ruthenium from rhodium. His prototypes for the two concepts are identical. So the concepts ought to be identical too, according to the prototype theory.

- The problem of irrelevant detail. Prototypes may include features that are not part of the concept. Fernando Torres is the prototype of a footballer. But FERNANDO TORRES is not part of the concept FOOTBALLER. If it were, then the concept FOOTBALLER would change as older footballers retire and younger footballers become famous.

- Psychological essentialism. Experimental evidence suggests that we use some concepts as if we are essentialists, thinking that the correct application of some concept is governed by some factor of which we may be unaware.

A natural conclusion to draw from these objections is that prototypicality structures are not constitutive of concepts. The very same evidence supports 
equally that claim that prototypicality structures are associated with concepts but not constitutive of them, and that concepts get their identity via some other mechanism. For example RUTHENIUM and RHODIUM get their identities by being hooked up to the world in different ways. If I learn more about RUTHENIUM, so that I now have a richer prototypicality structure, that is not a matter of acquiring a new concept but of associating new facts/beliefs to my pre-existing concept. The prototype theorist looks forced to accept something like this for PET FISH; the same is no less true of PET and FISH also. The graded structure revealed by experiments may best be understood not as revealing the facts about our concept but rather facts about the epistemology of applying those concepts. For example, we may use prototypes in the application of concepts, but those prototypes are just heuristic devices to enable faster processing.

It is a largely empirical matter whether the prototype theory can overcome such objections, and whether they also present insurmountable problems for the dynamic frames account. Even if we accept the broad approach defined by those theories, do such theories in fact help us understand what goes on in scientific revolutions? Is it the case, as $\operatorname{ABC}(1998,18)$ declare, that 'revisions in taxonomy .... are now the distinguishing feature of revolutions'. Are anomalies cases that cause tension in an existing conceptual structure since they violate hierarchical principles or demand divergent categorizations (1998, 7; 2006, 69-72)? I suggest that these claims are mistaken. Scientific revolutions are frequently accompanied by conceptual changes, and in some cases conceptual change may be central to the nature of the revolution. But in some cases there is no significant conceptual change, and even in the cases where there is conceptual change, that change is typically not all that there is to the scientific revolution. The principal reason for these claims is simple. Core to most science is belief. And in many cases to understand fully what happens in a revolution requires appreciation how beliefs changed. And not all belief change, even significant belief change, is conceptual change.

It is simple to find anomalies in the history of science that do not satisfy $\mathrm{ABC}$ 's description of them as violations of hierarchical principles, and which do not create pressure for categorisation of things or phenomena in diverse ways. Here are some examples:

- Anomalous planetary orbits. While Newton had been able to show that principal 'inequalities' in the motion of the Moon were due to the gravitational attraction of the Sun, nonetheless Newton's successors were unable to eliminate a significant discrepancy between the predictions of the theory and what was observed. In the 1740s the discrepancy was held by some to be an anomaly requiring possible adjustment to Newton's inverse square law (with further terms). The anomaly violates no principle of categorisation; it is a simple mismatch between what the theory demanded and what was observed. As it was, Clairaut was ultimately able to resolve the anomaly by correcting certain empirical approximations. But 
had the inverse square law required changing, that would not have been a change in taxonomy. Much the same can be said about the anomalous precession of the perihelion of Mercury. While the revolutionary general theory of relativity did involve conceptual revision, the anomaly in the orbit of Mercury did not itself imply any tension on categorisation or pose any threat to hierarchical principles.

- Anomalies in classical physics. (i) The ultraviolet (Rayleigh-Jeans) catastrophe. Classical physics predicts that a black-body in equilibrium will emit an infinite quantity of energy in any finite time. Clearly it does not. (ii) Models of the atom. The results of the Geiger-Marsden experiment were anomalous in the light of the then current 'plum-pudding' model of the atom (Thomson). Rutherford, following a suggestion of Nagaoka, proposed a concentration of positive charge in what we call the nucleus, with electrons forming a cloud. While resolving the preceding anomaly, because the nucleus is able to repel the alpha particle, this model created its own anomaly. For the electrons to remain at a distance from the nucleus they must be moving (like planets around the sun), but their motion would lead to loss of energy as electromagnetic radiation, leading them to spiral into the nucleus. Yet atoms are clearly stable. Both the latter anomaly and the ultraviolet catastrophe were resolved by the development of the quantum theory, which indeed involved important conceptual change. But as above, the anomalies themselves do not breach hierarchical principles or suggest divergent categorisations.

- Anomalies in Galen. Galen's human anatomy, much of which had been based on dissections of apes, came under critical scrutiny in the sixteenth and seventeenth centuries. Vesalius showed that many of Galen's assertions are not born out by observations of the human body. For example, Galen claimed that there is a porous interventricular septum, so that blood could pass from the right ventricle of the heart to the left (as his model required). Vesalius's dissections published in the second edition of De Humani Corporis Fabrica showed this to be false. This was not only a mistake in Galen's work, but was anomalous for his theory of the movement of the blood. Perhaps the best known anomaly for that theory is that expounded by Harvey, who in chapter eight of De Motu Cordis estimated that quantity of blood pumped by the heart (about $250 \mathrm{~kg}$ in a day). Galen's theory held that (venous) blood was produced by the liver and absorbed elsewhere in the body. But clearly it would be impossible for the liver to produce this quantity of new blood. The anomalies are significant for Galen's theory. But they are once again straightforward to understand: the dominant theory held or implied $p$; observation show that $p$ is false.

Such cases show that anomalies are not always cases that violate hierarchical principles; often they are simple (though significant) disagreements between 
theory and observation - in the physical sciences such disagreements may be simply quantitative and so no question of taxonomy need be raised.

Even if I am right about anomalies, it might nonetheless be the case that any resulting revolution is a significant change in taxonomy. Many examples, however, show that there are revolutions that do not result in significant taxonomic change. One significant problem with the $\mathrm{ABC}$ view is presented by science of which taxonomy is not a significant element.

- Einstein's special and general theories of relativity undoubtedly made revolutionary contributions to physics, yet the physics in question is not concerned with taxonomy. No doubt one can construct taxonomies that would be affected by these changes. So the relativity of simultaneity disrupts a taxonomy of events that is available under classical assumptions (e.g. 'past', 'present', 'future'). But such taxonomies are not central to the Einsteinian revolutions and to attempt to characterize the revolutions in terms of taxonomic change would be to miss the key innovations of Einstein's theories.

Kuhn $(1970,101-2)$ does argue that there is conceptual change in general relativity (concerning the terms 'space', 'time', and 'mass'). But these are not taxonomic terms. ABC's claim that revolutionary change is taxonomic change is the consequence of two assertions, that revolutionary change is conceptual change and that conceptual change is taxonomic change, which imposes a double straight-jacket on revolutions.

Even sciences with taxonomies can undergo revolutions that do not involve significant conceptual change and without disrupting taxonomic structures. Here are some examples:

- The discovery of the structure of DNA. One of the most far-reaching scientific discoveries of all time, Crick and Watson's elucidation of the structure of DNA must count as revolutionary in that it transformed biology and biochemistry and gave rise to several new scientific fields (such as molecular genetics). In so doing the discovery led to the addition of new taxonomic categories and indeed new taxonomies structures. Yet it did not require any radical changes to existing structures. The taxonomic effects are cumulative rather than revisionary.

- The cause of stomach ulcers. The standard view was that the principal cause of gastric ulcers is excess stomach acid, which could be brought about by factors such as stress. Barry Marshall and Robin Warren showed that $90 \%$ of such ulcers are caused by the bacterium $H$. pylori. This was a revolutionary change. It overturned a theory that had held sway for decades and which underpinned a raft of clinical procedures and commercial activities, including psychoanalytic therapies, surgery, and a multibillion dollar pharmaceutical industry. It was fiercely resisted for some time, but is now the accepted view, with corresponding changes in scientific and clinical practice. In this case there is a change in classification. 
We might have a classification of diseases by primary cause, and peptic ulcers (gastric and duodenal ulcers) have moved from 'stress-induced' to 'bacterial infection'. That change is not a change in taxonomic structure, just a change in where one places an item in an unchanged structure. Hence ABC's theory cannot account for it as a revolutionary change.

- The function of the heart. Harvey's response to the anomalies in Galen's account of the heart and blood was to provide a new theory of their function and motion: blood circulates, pumped by he heart. This is a radical departure from Galen's teaching and is righty regarded as one of the most important revolutionary discoveries in physiology, notwithstanding the fact that there are many continuities between Harvey's thinking and the preceding era. Harvey's work had a profound influence on subsequent physiology. For example, given that Harvey had shown that the liver does not create blood, then it is natural to ask what then is the function of the liver, thereby stimulating novel (and also revolutionary) work by Batholin and others on the liver and lymphatic system. Furthermore, Harvey's work was pioneering in terms of technique, as an exemplar of experimental physiology. It is difficult to see how this revolution can be characterised as a change in taxonomic structure.

- The discovery of nuclear structure. The two decades from 1909 saw a radical transformation in our understanding of the structure of the atom and in particular of the nucleus, with much of the work directed or inspired by Ernest Rutherford. As discussed above, the Geiger-Marsden experiment led to the development of the Rutherford-Bohr model of the atom, with positive charge concentrated in a 'nucleus'. Bohr's version of model end experimental work by Moseley implied a relationship between atomic number and nuclear charge, which in turn suggested that there are discrete entities each with unit positive charge, experimentally confirmed by Rutherford's 'splitting the atom' experiment. Yet this raised the question, how could discrete like charges be held together against their mutually repulsive forces, which led to the hypothesis of further, uncharged nuclear particles and the discovery of the neutron by Chadwick in 1932. This sequence of discoveries led to the science of nuclear physics, some important aspects of which are described in detail by ABC. Like the discovery of the structure of DNA, a principal contribution of this revolution is that is opens up a while new field of science, providing a paradigm of how that science is to be carried out. As as in the case of Harvey, the revolution involved and promoted the development of new experimental techniques, for example the use of high-energy particles to probe the structure of matter that became exemplars of experimental methods that have developed to the present day.

In most of these cases the revolutions are best understood as changes in what is believed, whose significance is generated by the theoretical and exper- 
imental context in which they occur. In addition others involve revolutionary changes in experimental technique. Kuhn explains, for example, how the discovery of X-rays was revolutionary because it potentially called into question the use of cathode ray tubes and the results produced by them, while also opening up a new field. The development of staining techniques in cytology and statistical tests in social research are further examples. Many cases in the history of science will exhibit theoretical and experimental change. In both kinds of case, new paradigms are generated: new exemplars of scientific thinking and doing. In these cases, I have argued, changes in conceptual structure are not significant in understanding what is going on. Some cases involved additions to conceptual structure, but for ABC such additions are not revolutionary changes, for the latter require disruption to conceptual structure. In other cases there may be some such disruption: arguably the concept of CHEMICAL ELEMENT went through a disruptive change as a result of the changes in understanding of atomic structure. Maybe with a little ingenuity one could make a case for some kind of conceptual change in all these cases. But any such changes would be tangential to the cognitive changes that science and scientists underwent. Since ABC make a general claim about the nature of scientific revolutions (as being a matter of taxonomic change), such cases serve to refute their thesis. Refuting their general thesis does not require asserting that the sort of conceptual change they describe is never central to a scientific revolution. Sometimes it may well be, and the cases they describe are excellent candidates. The conclusion we should draw from their cases is not that all revolutions involve such change but only that some revolutions involve such changes. We may add to the kinds of revolutionary changes in science mentioned in the first two sentences of this paragraph: depending on the scientific context (theoretical, technical, conceptual), a scientific revolution might be a significant change to what is believed, to experimental technique, or to conceptual structure. In each case the revolution will create new exemplars.

I shall now turn to incommensurability, albeit briefly, for my conclusions concerning incommensurability are corollaries of what has been said above. $\operatorname{ABC}(1998,6)$ tell us that 'the notion of incommensurability [is] the central theme of Kuhn's theory of scientific revolutions'. Incommensurability does not play a major role in Kuhn's theory of scientific revolutions as found in Structure (furthermore, the incommensurability there is methodological as well as conceptual). Kuhn does give incommensurability a central role in his later work. But since he did not undertake a concerted revision of his theory of scientific revolutions, it cannot be said that incommensurability becomes the central theme of that theory, whose principal source remains Structure. Secondly, the examples given above of revolutionary changes in science without taxonomic shifts are a fortiori examples of revolutionary change without taxonomic incommensurability. We noted that Kuhn himself argues that there is conceptual change and incommensurability in the Einsteinian case. If Kuhn is correct about that, then it isn't taxonomic incommensurability. Yet, for ABC, incommensurability is taxonomic incommensurability. 
If we start not from a theory of incommensurability, but from the phenomenon of incommensurability, matters look different. In Structure, we identify incommensurability by its symptoms, such as a sense that the world has changed, something like a Gestalt switch, when what seemed incoherent now makes sense. We can see how such phenomena did occur in some of these cases. For example, Barry Marshall's wife reports overhearing comments at a gastroenterology conference in the United States, 'They were talking about this terrible person that they imported from Australia to speak. You know: "How could they put such rubbish in the conference?"' (Schulz 2010). Despite the fact that bacterial infections are so common and the fact that no-one had any direct evidence against Marshall's theory, it was branded as rubbish. In part because physicians believed that stomachs were too acidic for bacteria (though bacteriologists knew better) and in large part because a totally different theory (the stress theory) had held sway for so long and had informed every aspect of their thinking and practice, it was difficult for them to see that such a radical alternative could be scientifically respectable. This, I suggest, is an important phenomenon in understanding scientific change, but it is one not one captured by thinking in terms of conceptual change. It is nonetheless, I suggest, one that can be readily understood in terms of cognitive habits.

\section{Conclusion}

ABC make bold claims about their approach to scientific revolutions and incommensurability. They elaborate Barsalou's dynamic frame account of concepts and assert 'We will show that all of the important features of Kuhn's model [of scientific revolutions] may now be seen as consequences of this fundamental account of human concepts and its dynamics' $(1998,6)$. I think this is badly mistaken. For a start, we should be alive to problems with ABC's preferred approach to concepts and the fact that it has competitors. Be that as it may, does such a theory provide us with a way of capturing Kuhn's theory of scientific change? No, because conceptual change is not central to his theory as articulated in Structure; incommensurability is not a central theme of Structure. The references to Wittgenstein are late additions and incidental to his theory. And they are not intended to articulate a theory of concepts; they are intended to be an example of learning similarity relations without learning explicit rules. The focus of Structure is paradigms, both regarded as shared commitments of scientific community and as a particular set of commitments, the shared exemplars. The latter constitute Kuhn's most significant innovation, and are, I suggest, best understood with the tools of cognitive psychology, in particular with the aid of research on pattern recognition, analogical thinking, and case-based reasoning. What I say about Structure may be consistent with what ABC say about Kuhn, if Kuhn developed a later theory of scientific revolutions, one radically different from that in Structure. But they present no compelling evidence that he did. 
Kuhn did develop a later theory of taxonomic incommensurability, and ABC's scholarship is very helpful in showing how the frame theory of concepts may illuminate and develop that theory. Since incommensurability is not central to Kuhn's account of scientific revolutions, this fact does not license the bold claim the 'all of the important features of Kuhn's model' are consequences of the frame account of concepts. Rather, what $\mathrm{ABC}$ have done is show how the frame account and taxonomic incommensurability may be central elements of some scientific revolutions and parts (but not the only parts) of other scientific revolutions; we must note that they may also be absent from some scientific revolutions. That important restriction on what the frame account can do matches the fact that we can find anomalies and scientific revolutions that simply do not fit their model.

To the historical examples adduced to support the last claim, at least as regard revolutions, two responses are conceivable. The first will say that my examples of revolutions are not true Kuhnian revolutions. For example, the revolutions ensuing from the discoveries of the structure of DNA and of the structure of the atomic nucleus are not revolutions because they are not revisionary. Or the discovery of the cause of stomach ulcers is not revolutionary because it is small scale. Such responses would not be true to Kuhn's aims. Kuhn is explicit that although the most important characteristics of scientific revolutions emerge from study of grand revolutions (such as the Newtonian or chemical revolutions), 'It is ... a fundamental thesis of this essay [structure] that they can also be retrieved from the study of many other episodes that were not so obviously revolutionary' (Kuhn 1970, 6). Non-revisionary changes often do involve competition between often radically different theories and the development of new paradigms and exemplars, and they can show incommensurability. And Kuhn himself came to regard the development of new specialties as important, seeing the process as analogous to speciation.

In any case, I see no reason why important revisionary changes should necessarily be accompanied by taxonomic changes. Not all science involves taxonomy. Not all changes in belief imply a change of taxonomy, even where the latter is present. An important change in the transition from Aristotelian to Newtonian physics is the move from thinking that all motion requires explanation to thinking that it is only non-uniform motion that needs explaining; that does not look as if it can be neatly explained as a taxonomic change. Furthermore, scientific revolutions can centre on changes in practice and technique, and these are even further removed from taxonomic change. ABC $(2006,33)$ do note, it should be acknowledged, that it is a possible shortcoming of their account that it provides only limited insight into 'nomic' concepts. Normic concepts are those acquired though learning similarity (and difference) relations by ostension; these are the concepts to which their theory applies. Nomic concepts are acquired via the complex problem situations in which the concept and the law in which it figures are applied. ABC say that Kuhn did not give an account of how to identify the referents of individual nomic concepts in such cases. This restriction on the application of their theory is potentially very 
significant. For it could be argued that very few scientific concepts are normic by this characterisation, since not many scientific concepts are introduced by ostension (the concepts discussed in their example of nuclear physics would be excluded by this criterion). ABC say it would be hubristic to claim a complete account of all scientific concepts; if so, then they are not in a position to claim to give a general account of scientific change. As it is, I think that accepting limitations on the reach of their theory is the correct response.

The second kind of response aims to maintain much of the universality of ABC's claim by being liberal with what counts as taxonomic change. In particular, they might argue that I have not appreciated the significance of the 'constraints' that operate between taxonomic categories. Beliefs act as such constraints and so revisions to such beliefs do lead to taxonomic change. The danger with this approach is that is makes the theory less informative while also diverting the focus. Too much counts as conceptual change (any belief change has that effect). More importantly, let us imagine that we can come up with Aristotelian and Newtonian frames such that the explanatory shift mentioned above can be represented as a conceptual change. How would that help us understand what is important about that shift? Would that explain, for example, why Galileo's physics (proto-Newtonian in this regard) was difficult for many Aristotelians to understand fully?

It strikes me that the undue emphasis on conceptual change is a hangover, albeit in an up-to-date and scientifically well-informed guise, of the linguistic approach to philosophy. For several decades many analytic philosophers thought that philosophical problems were always linguistic in character. Now this is not a widespread view, especially as naturalism has become more common in philosophy. Appealing to a sophisticated account of concepts from cognitive science is a way of working within the naturalistic paradigm while hanging onto the older conviction that language is all. But it is not everything, not even in cognitive science. So while we should welcome the insights offered by $\mathrm{ABC}$ we should not regard those insights as explaining everything that Kuhn wanted to explain; to do so is to adopt the same procrustean approach offered by the old linguistic philosophy. Rather those insights should be deployed alongside other discoveries and theories in cognitive science that are not conceptual in focus, for example the work on analogy, case based reasoning, cognitive habits, and quasi-intuitive connections that I sketched above in section 2. With a broader set of explanatory tools, I believe that we can come closer to showing how cognitive science can vindicate many of Kuhn's most interesting claims in The Structure of Scientific Revolutions. 


\section{REFERENCES}

Andersen, H., P. Barker, and X. Chen. 1996. Kuhn's mature philosophy of science and cognitive psychology. Philosophical Psychology 9: 347-63.

Andersen, H., P. Barker, and X. Chen. 2006. The cognitive structure of scientific revolutions. Cambridge: Cambridge University Press.

Bird, A. 2005. Naturalizing Kuhn. Proceedings of the Aristotelian Society 105: 109-27.

Bird, A. 2007. Incommensurability naturalized. In Rethinking Scientific Change and Theory Comparison, edited by L. Soler, H. Sankey, and P. Hoyningen-Huene, Volume 255 of Boston Studies in the Philosophy of Science, 21-39. Dordrecht: Spinger.

Carnap, R. 1956. The methodological character of theoretical concepts. Minnesota Studies in the Philosophy of Science 1: 38-76.

Chen, X., H. Andersen, and P. Barker. 1998. Kuhn's theory of scientific revolutions and cognitive psychology. Philosophical Psychology 11: 5-28.

Chi, M. T. H., P. J. Feltovich, and R. Glaser. 1981. Categorization and representation of physics problems by experts and novices. Cognitive Science 5: 121-52.

Dummett, M. 1991. The logical basis of metaphysics. London: Duckworth.

Dunbar, K. 1996. How scientists really reason. In The Nature of Insight, edited by R. Sternberg and J. Davidson, 365-95. Cambridge, MA: MIT Press.

Dunbar, K. 1999. How scientists build models: In vivo science as a window on the scientific mind. In Model-Based Reasoning in Scientific Discovery, edited by L. Magnani, N. J. Nersessian, and P. Thagard, 85-99. New York, NY: Kluwer/Plenum.

Gallie, W. B. 1955. Essentially contested concepts. Proceedings of the Aristotelian Society 56: 167-98.

Gentner, D., K. Holyoak, and B. Kokinov. 2001. The analogical mind: Perspectives from cognitive science. Cambridge MA: MIT Press.

Gentner, D., and M. Jeziorski. 1993. The shift from metaphor to analogy in western science. In Metaphor and Thought (2nd ed.), edited by A. Ortony, 447-80. Cambridge: Cambridge University Press.

Grosvenor, B. 2011. On the importance of connoisseurship. Fine Art Connoisseur 8: 50-2.

Hanson, N. 1958. Patterns of discovery: An inquiry into the conceptual foundations of sciences. Cambridge: Cambridge University Press.

Hart, H. L. A. 1961. The concept of law. Oxford: Clarendon Press.

Holyoak, K. J., and P. Thagard. 1995. Mental leaps: Analogy in creative thought. Cambridge, MA: MIT Press.

Holyoak, K. J., and P. Thagard. 1997. The analogical mind. American Psychologist 52: $35-44$.

Hoyningen-Huene, P. 2006. More letters by Paul Feyerabend to Thomas S. Kuhn on ProtoStructure. Studies in History and Philosophy of Science 37: 610-32.

Jastrow, J. 1899. The mind's eye. Popular Science Monthly 54: 299-312.

Kindi, V. 1995a. Kuhn and Wittgenstein: Philosophical investigation of the structure of scientific revolutions. Athens: Smili editions.

Kindi, V. P. 1995b. Kuhn's The structure of scientific revolutions revisited. Journal for General Philosophy of Science 26: 75-92.

Kripke, S. 1971. Identity and necessity. In Identity and Individuation, edited by M. K. Munitz. New York, NY: New York University Press.

Kripke, S. 1980. Naming and necessity. Oxford: Blackwell. 
Kuhn, T. S. 1970. The Structure of Scientific Revolutions, 2nd ed. Chicago, IL: University of Chicago Press.

Kuhn, T. S. 1974. Second thoughts on paradigms. In The Structure of Scientific Theories, edited by F. Suppe, 459-82. Urbana: University of Illinois Press. Page references to reprint in Kuhn 1977.

Kuhn, T. S. 1977. The essential tension. Chicago, IL: University of Chicago Press.

Kuhn, T. S. 1987. What are scientific revolutions? In The Probabilistic Revolution, edited by L. Krüger, L. J. Daston, and M. Heidelberger, 7-22. Cambridge: Cambridge University Press.

Kuhn, T. S. 1991. The road since Structure. In PSA 1990, edited by A. Fine, M. Forbes, and L. Wessels, Volume 2, 2-13. East Lansing, MI: Philosophy of Science Association.

Kuhn, T. S. 1993. Afterwords. In World Changes. Thomas Kuhn and the Nature of Science, edited by P. Horwich, 311-41. Cambridge, MA: MIT Press.

Laurence, S., and E. Margolis. 1999. Concepts and cognitive science. In Concepts: Core Readings, edited by S. Laurence and E. Margolis, 3-81. Cambridge MA: MIT Press.

MacIntyre, A. 1973. The essential contestability of some social concepts. Ethics 84(10): 1-9.

Mandelbaum, M. 1965. Family resemblances and generalization concerning the arts. American Philosophical Quarterly 2: 219-228.

Marcus, R. B. 1961. Modalities and intensional languages. Synthese 13: 303-22.

Margolis, H. 1987. Patterns, thinking, and cognition. A theory of judgment. Chicago, IL: University of Chicago Press.

Nagel, E. 1961. The structure of science: Problems in the logic of scientific explanation. New York: Harcourt, Brace \& World.

Narboux, J.-P. 2003. Incommensurabilité et exemplarité. Archives de Philosophie 66: 43747.

Nersessian, N. 1987. A cognitive-historical approach to meaning in scientific theories. In The Process of Science, edited by N. Nersessian, 161-77. Dordrecht: Kluwer.

Nersessian, N. 1992. How do scientists think? Capturing the dynamics of conceptual change in science. Cognitive Models of Science 15: 3-44.

Nersessian, N. 2003. Kuhn, conceptual change, and cognitive science. In Thomas Kuhn, edited by T. Nickles, 179-211. Cambridge: Cambridge University Press.

Nickles, T. 2003. Normal science: From logic to case-based and model-based reasoning. In Thomas Kuhn, edited by T. Nickles, 142-77. Cambridge: Cambridge University Press.

Quine, W. V. 1951. Two dogmas of empiricism. The Philosophical Review 60: 20-43.

Quine, W. V. 1960. Word and object. Cambridge MA: MIT Press.

Read, R. 2005. How and how not to write on a "legendary" philosopher. Philosophy of the Social Sciences 35: 369-87.

Rosch, E. 1973. On the internal structure of perceptual and semantic categories. In Cognitive Development and the Acquisition of Language, edited by T. E. Moore, 111-44. New York NY: Academic.

Rosch, E. 1988. Principles of categorization. In Readings in Cognitive Science, a Perspective from Psychology and Artificial Intelligence, edited by A. Collins and E. E. Smith, 312-22. San Mateo CA: Morgan Kaufmann Publishers.

Rosch, E., and C. B. Mervis. 1975. Family resemblances: Studies in the internal structure of categories. Cognitive Psychology 7: 573-605.

Sankey, H. 1998. Taxonomic incommensurability. International Studies in the Philosophy of Science 12: 7-16.

Theoria 75 (2012): 293-321 
Schlick, M. 1918. Allgemeine Erkenntnislehre. Berlin: Julius Springer.

Schulz, K. 2010. Stress doesn't cause ulcers! Or, how to win a Nobel Prize in one easy lesson: Barry Marshall on being ...right. http://www.slate.com/blogs/thewrongstuff/ 2010/09/09/stress_doesn_t_cause_ulers_or_how_to_win_a_nobel_prize_in_one easy_lesson_barry_marshall_on_being_right.html accessed $2 \overline{2}$ June 2012.

Sharrock, W., and R. Read. 2002. K Kuhn: Philosopher of scientific revolution. Cambridge: Polity.

von Wright, G. H. 1963. The varieties of goodness. London: Routledge and Kegan Paul. Weitz, M. 1956. The role of theory in aesthetics. The Journal of Aesthetics and Art Criticism 15: 27-35.

Alexander BIRD is Professor of Philosophy at the University of Bristol. The is the author of Philosophy of Science (1998), Thomas Kuhn (2000), and Nature's Metaphysics: Laws and Properties (2007). With James Ladyman he was editor of the British Journal for the Philosophy of Science between 2004 and 2011.

ADDRESS: Department of Philosophy, Bristol University, 9 Woodland Rd, Bristol BS8 1TB, UK.

E-mail: Alexander.Bird@bristol.ac.uk 